\title{
New regular partial difference sets and strongly regular graphs with parameters $(96,20,4,4)$ and $(96,19,2,4)$
}

\author{
Anka Golemac, Joško Mandić and Tanja Vučičić \\ University of Split, Department of Mathematics \\ Teslina 12/III, 21000 Split, Croatia \\ golemac@pmfst.hr, majo@pmfst.hr, vucicic@pmfst.hr
}

Submitted: Sep 15, 2005; Accepted: Sep 29, 2006; Published: Oct 19, 2006

Mathematics Subject Classification: 05B05, 05B10, 05E30

\begin{abstract}
New $(96,20,4,4)$ and $(96,19,2,4)$ regular partial difference sets are constructed, together with the corresponding strongly regular graphs. Our source are $(96,20,4)$ regular symmetric designs.
\end{abstract}

Keywords: Difference set, partial difference set, Cayley graph, symmetric design.

\section{Introduction and preliminaries}

We start with defining objects to be constructed.

Definition 1 Let $H$ be a group of order $v$. A $k$-subset $S \subset H$ is called a $(v, k, \lambda, \mu)$ partial difference set if the multiset $\left\{x y^{-1} \mid x, y \in S, x \neq y\right\}$ contains each nonidentity element of $S$ exactly $\lambda$ times and it contains each nonidentity element of $H \backslash S$ exactly $\mu$ times.

Using the notation of a group ring $\mathbb{Z} H$ (where $\underline{S}:=\sum_{s \in S} s$ ), a $(v, k, \lambda, \mu)$ partial difference set $S \subset H$ in the group $H$ can be defined as a subset for which the equation

$$
\underline{S} \cdot \underline{S^{(-1)}}=k \underline{\{e\}}+\lambda \underline{S \backslash\{e\}}+\mu \underline{(H \backslash S) \backslash\{e\}}
$$

holds; $e$ denotes the group identity element.

Partial differential sets $S_{1}$ and $S_{2}$ in groups $H_{1}$ and $H_{2}$, respectively, we will call equivalent if there exists a group isomorphism $\varphi: H_{1} \rightarrow H_{2}$ which maps $S_{1}$ onto $S_{2}$.

The notion of a partial difference set generalizes that of a difference set, well-known in group and design theory. 
Definition $2 A(v, k, \lambda)$ difference set is a $k$-element subset $\Delta \subseteq H$ in a group $H$ of order $v$ provided that the multiset $\left\{x y^{-1} \mid x, y \in \Delta, x \neq y\right\}$ contains each nonidentity element of $H$ exactly $\lambda$ times.

In terms of a group ring, $\Delta \subseteq H$ is a difference set in a group $H$ if and only if the relation $\underline{\Delta} \cdot \underline{\Delta^{(-1)}}=k\{e\}+\lambda H \backslash\{e\}$ holds in $\mathbb{Z} H$. In case a set $\Delta \subseteq H$ is a difference set in a group $H$, its so called "shift" $\Delta x$ by each element $x \in H$ is a difference set in $H$ as well, [1]. It is obvious that any $(v, k, \lambda)$ difference set is a $(v, k, \lambda, \lambda)$ partial difference set.

A partial difference set (PDS for short) $S$ is reversible if $S=S^{(-1)}$. A reversible partial difference set $S$ is called regular if $e \notin S$. It is easy to see (cf. [7]) that the following assertions hold.

Proposition 1 Suppose that $S$ is a reversible $(v, k, \lambda, \mu) P D S$ in a group $H$, such that $e \in S$. Then $(S-e)$ is a regular $(v, k-1, \lambda-2, \mu) P D S$ in $H$. Conversely, if $S$ is a regular $P D S$ in $H$, then $(S+e)$ is a reversible $P D S$ with corresponding parameters.

Proposition 2 Suppose that $\Delta$ is a $(v, k, \lambda)$ difference set in $H, x \in H$. Then

(i) $\Delta x$ is a regular $(v, k, \lambda, \lambda) P D S$ if and only if $x^{-1} \notin \Delta$ and $\Delta x$ is a reversible set;

(ii) $\Delta x-e$ is a regular $(v, k-1, \lambda-2, \lambda) P D S$ if and only if $x^{-1} \in \Delta$ and $\Delta x$ is a reversible set.

The development of a difference set $\Delta \subseteq H$ is the incidence structure dev $\Delta=$ $(H,\{\Delta g \mid g \in H\}, \in)$. By this structure difference sets and symmetric designs are interrelated, as shows the following important result, [1].

Theorem 1 Let $H$ be a finite group of order $v$ and $\Delta$ a proper, non-empty k-element subset of $H$. Then $\Delta$ is a $(v, k, \lambda)$ difference set in $H$ if and only if dev $\Delta$ is a symmetric $(v, k, \lambda)$ design on which $H$ acts regularly.

Let's repeat, a symmetric block design with parameters $(v, k, \lambda)$ is a finite incidence structure $\mathcal{D}=(\mathcal{V}, \mathcal{B}, \mathcal{I})$ consisting of $|\mathcal{V}|=v$ points and $|\mathcal{B}|=v$ blocks, where each block is incident with $k$ points and any two distinct points are incident with exactly $\lambda$ common blocks. An automorphism of a symmetric block design $\mathcal{D}$ is a permutation on $\mathcal{V}$ which sends blocks to blocks. The set of all automorphisms of $\mathcal{D}$ forms its full automorphism group denoted by $A u t \mathcal{D}$. If a subgroup $H \leq A u t \mathcal{D}$ acts regularly on $\mathcal{V}$ and $\mathcal{B}$, then $\mathcal{D}$ is called regular and $H$ is called a Singer group of $\mathcal{D}$.

Two difference sets $\Delta^{1}$ (in $H^{1}$ ) and $\Delta^{2}$ (in $H^{2}$ ) are isomorphic if the designs dev $\Delta^{1}$ and dev $\Delta^{2}$ are isomorphic; $\Delta^{1}$ and $\Delta^{2}$ are equivalent if there exists a group isomorphism $\varphi: H^{1} \rightarrow H^{2}$ such that $\varphi\left(\Delta^{1}\right)=\Delta^{2} g$ for a suitable $g \in H^{2}$. It is easy to see that equivalent difference sets $\Delta^{1}$ and $\Delta^{2}$ give rise to isomorphic symmetric designs dev $\Delta^{1}$ and dev $\Delta^{2}$. Depending on the respective property of $H$, a difference set (and PDS as well) is called abelian, cyclic, or nonabelian.

The so far introduced notions and observations are connected to graph theory. More precisely, regular partial difference sets and strongly regular graphs are closely related through the concept of Cayley graphs. 
Definition $3 A$ strongly regular graph (SRG) with parameters $(v, k, \lambda, \mu)$ is a graph with $v$ vertices which is regular of valency $k$, i.e. every vertex is incident with $k$ edges, such that any pair of adjacent vertices have exactly $\lambda$ common neighbours and any pair of non-adjacent vertices have exactly $\mu$ common neighbours.

Definition 4 For a group $H$ and a set $S \subset H$ with the property that $e \notin S$ and $S=S^{(-1)}$, the Cayley graph $\Gamma=C a y(H, S)$ over $H$ with connection set $S$ is the graph with vertex set $H$ so that the vertices $x$ and $y$ are adjacent if and only if $x^{-1} y \in S$. Then $\Gamma$ is undirected graph without loops.

Accordingly, the edge set of a Cayley graph $\Gamma=C a y(H, S)$ over $H$ with connection set $S$ is $E:=\{\{x, s x\} \mid x \in H, s \in S\}$. Our construction of strongly regular graphs (cf. [5]) will be based on the following important assertion about Cayley graphs, [1] p. 230 or $[6]$.

Theorem 2 A Cayley graph $C a y(H, S)$ is a $(v, k, \lambda, \mu)$ strongly regular graph if and only if $S$ is a $(v, k, \lambda, \mu)$ regular partial difference set in $H$.

In this sense, equivalent regular PDS's obviously correspond to isomorphic strongly regular Cayley graphs. Note that for two inequivalent partial difference sets $S_{1}$ and $S_{2}$ in a group $H$, the graphs $\operatorname{Cay}\left(H, S_{1}\right)$ and $\operatorname{Cay}\left(H, S_{2}\right)$ can be isomorphic. Similarly, for two inequivalent partial difference sets $S_{1}$ and $S_{2}$ in groups $H_{1}$ and $H_{2},\left|H_{1}\right|=\left|H_{2}\right|$, the graphs $\operatorname{Cay}\left(H_{1}, S_{1}\right)$ and $\operatorname{Cay}\left(H_{2}, S_{2}\right)$ can be isomorphic. Several examples of both such cases are shown in Section 2.

In our computation we use GAP, the well-known system for computational group theory, [9]. Moreover, because we deal with a rather large number of groups, for identifying groups we use GAP-catalogue number whenever it is available. Namely, the order of some groups that appear in our considerations exceeds the scope of the GAP Library Small Groups. A GAP-catalogue number is of the form $[m, n]$ and it stands for $n$-th group of order $m$ in the catalogue. For graph exploring we use GRAPE [8], a package which is a part of GAP.

\section{Construction of regular partial difference sets and graphs}

Following the theoretical background highlighted in Section 1, it can easily be verified that the procedure for the search of regular partial difference sets, starting from a known difference set $\Delta \subseteq H$, can be performed in the next two steps:

(i) construction of all shifts $\Delta x$ of $\Delta, x \in H$,

(ii) selection of those shifts which are reversible sets in $H$.

Then, each reversible shift which does not contain $e$ is a regular $(v, k, \lambda, \lambda) \operatorname{PDS}$, while each reversible shift that contains $e$ yields a regular $(v, k-1, \lambda-2, \lambda) \operatorname{PDS} \Delta x-e$. 
To this procedure of "surveyed shifting" we have submitted about seventy $(96,20,4)$ difference sets in approximately 30 groups presented in [3] and [4]. The cited papers contain detailed description of the difference sets construction from 9 regular $(96,20,4)$ symmetric designs. The procedure ended in construction of 59 regular PDS's in 9 groups. After GAP-testing on group automorphisms, final result boiled down to 31 inequivalent regular PDS in 9 groups. Regarding isomorphism of the corresponding strongly regular Cayley graphs, these 31 PDS's split into eight nonisomorphic SRG-classes. Their representatives we denote by $\Gamma_{j}, j=1,2, \ldots, 8$. It turned out that difference set shifts being or yielding regular PDS's are connected with three designs only, all three given in [3] and there denoted by $D_{1}, D_{6}$, and $D_{8}$. Sticking to that labelling, in presentation of the obtained regular PDS's we indicate the originating design in the superscript of a concerned difference set $\Delta_{[96, n]}^{k}, k \in\{1,6,8\}$. The subscript refers to GAP-cn of the host group. The results we give group by group. All nine groups are nonabelian. We use the notation $p^{q}=q p q^{-1}$ for $p, q$ arbitrary elements of a group.

1. In the group:

$$
\begin{gathered}
H_{[96,64]}=\langle x, y, z, a| x^{4}=y^{4}=z^{2}=[x, y]=1, x^{z}=y, y^{z}=x, a^{3}=1, \\
\left.x^{a}=x^{-1} y^{-1}, y^{a}=x, a^{z}=a^{-1}\right\rangle
\end{gathered}
$$

two nonisomorphic difference sets enable construction of two inequivalent regular PDS's.

The shift of

$$
\begin{aligned}
\Delta_{[96,64]}^{1}= & 1+a^{2} x^{2} z+a^{2} x^{2} a x a z+a x a^{2} x^{2} z+a x a x+a^{2} x z+x^{2} a x a z+ \\
& x^{2} a x z+a x^{2} a^{2} x+x a^{2} z+x a x z+x^{2} a x a+a^{2} x a x z+a x a^{2} z+a x a x^{2} a z+ \\
& a x a^{2} x^{2}+a x^{2} a x+a^{2} x a x^{2}+x^{2} a^{2}+a x^{2} z
\end{aligned}
$$

by $a x a^{2} x^{2} z$, e extracted, is a regular PDS. The corresponding SRG with parameters $(96,19,2,4)$ we denote by $\Gamma_{1}$. Using GRAPE one finds $\left|A u t \Gamma_{1}\right|=9216$.

The shift of

$$
\begin{aligned}
\Delta_{[96,64]}^{8}= & 1+x^{2} a x a z+a x^{2} a x a+x a^{2} z+a^{2} x^{2} a x z+x^{3} z+x z+ \\
& a x^{2} a^{2} x z+x^{2} a^{2} z+x^{2} a^{2} x z+x^{3}+a x a^{2} x^{2}+x a x z+x^{2} a x+a x^{2} z+ \\
& a^{2} x^{2} a x a+a^{2} x a x^{2} a+a^{2} x^{2} a^{2} x z+x a z+a x^{2}
\end{aligned}
$$

by $a^{2} x a x^{2} a$ is a regular PDS. The corresponding SRG with parameters $(96,20,4,4)$ we denote by $\Gamma_{8},\left|A u t \Gamma_{8}\right|=138240$.

2. In the group:

$$
\begin{gathered}
H_{[96,70]}=\langle x, y, z, t, a| x^{2}=y^{2}=z^{2}=t^{2}=[x, y]=[x, z]=[x, t]=[y, z]=1, \\
\left.[y, t]=[z, t]=a^{6}=1, x^{a}=y t, y^{a}=x z, z^{a}=x y z, t^{a}=y z t\right\rangle
\end{gathered}
$$

two nonisomorphic difference sets enable construction of three inequivalent regular PDS's.

The shifts of

$$
\begin{aligned}
\Delta_{[96,70]}^{1}= & 1+a^{2} x+x a y a^{2}+a^{3} x a+a y+a^{4} x a y a^{2}+a^{5} x a^{2}+a^{5} x y a+a^{5} y a+ \\
& a x a+a^{5} x a+a^{5} x a y a^{2}+a^{2} x a^{2}+a^{2} x a^{2} y+a^{3} x a y+x a^{2} y a+ \\
& a^{4} x a^{2} y a^{2}+a^{2} x a y+y a+a^{3}
\end{aligned}
$$

by $a^{3} x a y$ and $a^{2} x a^{2}$, e extracted, are regular PDS's. Using GAP they are checked to be equivalent. The corresponding SRG's with parameters $(96,19,2,4)$ are isomorphic to $\Gamma_{1}$. 
The shift of

$$
\begin{aligned}
\Delta_{[96,70]}^{8}= & 1+a^{3} x a^{2} y a+a^{5} x a+a^{4} x a y a+a y+a x y+x a+a^{5} x a^{2}+a^{2} x a+ \\
& a^{5} x a^{2} y a^{2}+a^{2} y a+a^{2} x a y+a^{4} x a^{2} y a^{2}+a^{2}+x a^{2}+a^{5} x a y a^{2}+ \\
& a^{3} x y a^{2}+a^{5} x y+a^{4} y a+a^{5}
\end{aligned}
$$

by $a^{4} x a^{2} y a^{2}$ is a regular PDS. The corresponding SRG with parameters $(96,20,4,4)$ is isomorphic to $\Gamma_{8}$.

$\Delta_{[96,70]}^{8}$ multiplied by $x a^{2}$ gives another regular PDS, not isomorphic to the previous one. The corresponding SRG with parameters $(96,20,4,4)$ we denote by $\Gamma_{7}$. GRAPE reveals $\left|A u t \Gamma_{7}\right|=3072$.

Now we already have examples of pairs of inequivalent regular PDS's that correspond to isomorphic strongly regular Cayley graphs: $\Delta_{[96,64]}^{1} a x a^{2} x^{2} z \backslash\{e\}$ and $\Delta_{[96,70]}^{1} a^{3} x a y \backslash$ $\{e\}$ (or $\Delta_{[96,70]}^{1} a^{2} x a^{2} \backslash\{e\}$ ); $\Delta_{[96,64]}^{8} a^{2} x a x^{2} a$ and $\Delta_{[96,70]}^{8} a^{4} x a^{2} y a^{2}$. More examples follow.

3. In the group:

$$
\begin{gathered}
H_{[96,71]}=\langle x, y, z, a| x^{4}=y^{4}=z^{2}=[x, y]=1, x^{z}=x y^{2}, y^{z}=x^{2} y^{-1}, \\
\left.a^{3}=1, x^{a}=x^{-1} y^{-1}, y^{a}=x,[a, z]=1\right\rangle
\end{gathered}
$$

two nonisomorphic difference sets enable construction of two inequivalent regular PDS's.

The shift of

$$
\begin{aligned}
\Delta_{[96,71]}^{1}= & 1+a x^{2} a z+a^{2} x^{2} a x+x a x+x a x^{2} a+x a^{2} z+a^{2} x z+a x^{2} a^{2} z+a^{2} x a x+ \\
& a x a x^{2} z+a x a^{2} z+a x a x^{2}+a^{2} x a^{2}+a^{2} x a x^{2} a+a^{2} x a^{2} x^{2}+a x^{2} a^{2} x z+ \\
& a x^{2} a+a x a^{2} x z+a x^{2} a x+a x^{2} a x a,
\end{aligned}
$$

by $a x a x^{2}, e$ extracted, is a regular PDS. The corresponding SRG with parameters $(96,19,2,4)$ is isomorphic to $\Gamma_{1}$.

The shift of

$$
\begin{aligned}
\Delta_{[96,71]}^{8}= & 1+a x^{2} a^{2} x z+a^{2} x^{2} a x+x^{2} z+a x a x^{2} z+x a^{2} x z+x^{2} a x a z+a x a z+ \\
& a^{2} x a x z+a x^{2} a x a z+a x^{2} a x a+a^{2} x a x+a x^{2}+x a x^{2}+x^{2} a z+ \\
& a^{2} x a^{2} x z+a x z+x a x+a^{2} x a^{2} x^{2} z+x^{2} a x
\end{aligned}
$$

by $a x^{2}$ is a regular PDS. The corresponding SRG with parameters $(96,20,4,4)$ is isomorphic to $\Gamma_{8}$.

4. In the group:

$$
\begin{aligned}
& H_{[96,186]}=S_{4} \times C_{4}=\langle x, y, z, a| x^{4}=y^{2}=z^{2}=[x, y]=[x, z]=(z y)^{3}=1 \text {, } \\
& \left.a^{3}=1,[a, x]=1,(z a)^{2}=(z y a)^{2}=1\right\rangle
\end{aligned}
$$

a single difference set enables construction of only one, up to equivalency, regular PDS.

The shifts of

$$
\begin{aligned}
\Delta_{[96,186]}^{1} & =1+a^{2} x^{2}+a x^{3} y a y+a x y a^{2} y+a y+a x^{2} y+a^{2} x^{2} y a^{2} y a y+a x^{2} y a y+ \\
& x^{3} y a^{2} y a y+a x^{3} y a^{2} y a y+x y a+a^{2} x y a^{2}+a^{2} x+y+a y a+a y a^{2}+ \\
& a y a^{2} y+x^{3}+a^{2} y a^{2} y a y+a^{2} y
\end{aligned}
$$

by $a y a^{2} y$ and $a x^{2} y a^{2} y$ are equivalent regular PDS's. The corresponding isomorphic SRG's with parameters $(96,20,4,4)$ are denoted by $\Gamma_{2}$. Using GRAPE we checked $\left|A u t \Gamma_{2}\right|=$ 11520 . 
5. In the group:

$$
H_{[96,190]}=\left\langle x, y, a \mid x^{8}=y^{2}=1, x^{y}=x^{5}, a^{3}=1,(x a)^{2}=1,[y, a]=1\right\rangle
$$

two nonisomorphic difference sets enable construction of three inequivalent regular PDS's.

The shifts of

$$
\begin{aligned}
\Delta_{[96,190]}^{1} & =1+a x^{2} a^{2} x y+a x^{2} a^{2} y+a x^{4}+a^{2} x a^{2} y+x^{3} y+a^{2} y+a x a^{2}+ \\
& a^{2} x a^{2} x+a^{2}+a x a^{2} x^{2} y+x a^{2} x a y+a+x^{2} a y+a x a^{2} x a^{2} y+a^{2} x^{3}+ \\
& x y+a x^{3} a^{2} y+a^{2} x^{2}+y
\end{aligned}
$$

by $a^{2} y$ and $a^{2} x^{4} y$ are equivalent regular PDS's. The corresponding SRG's with parameters $(96,20,4,4)$ are isomorphic to $\Gamma_{2}$.

The shift of

$$
\begin{gathered}
\Delta_{[96,190]}^{8}=1+a^{2} x^{3} a y+x a^{2} x a^{2} y+a x^{3} y+a^{2} x a^{2}+a x^{3} a y+a^{2} x a^{2} y+ \\
a x^{3} a+a^{2} x^{2} a^{2} x y+a x y+a x a^{2} x a y+a x^{2} a^{2}+a x^{3} a^{2}+ \\
a^{2} x^{4} y+x a^{2} x a y+x a y+a x^{2} a+a x a^{2} x+a x a^{2} x^{2}+x^{3} a y
\end{gathered}
$$

by $x^{2} a^{2} y$ is a regular PDS. The corresponding SRG with parameters $(96,20,4,4)$ is isomorphic to $\Gamma_{8}$.

$\Delta_{[96,190]}^{8} a x a^{2} x y$ is another regular PDS, not isomorphic to the previous one. The corresponding SRG with parameters $(96,20,4,4)$ is isomorphic to $\Gamma_{7}$.

6. In the group:

$$
\begin{gathered}
H_{[96,195]}=\langle x, y, z, t, w, a| x^{2}=y^{2}=z^{2}=t^{2}=[x, y]=[x, z]=[x, t]=1, \\
{[y, z]=[y, t]=[z, t]=1, w^{2}=a^{3}=(w a)^{2}=1, x^{w}=y t} \\
\left.y^{w}=x z, z^{w}=t, t^{w}=z, x^{a}=x z, y^{a}=y t, z^{a}=t, t^{a}=z t\right\rangle
\end{gathered}
$$

seven inequivalent difference sets originating from three nonisomorphic symmetric designs enable construction of 12 inequivalent regular PDS's. Here Aut $D_{1}$ and $A u t D_{6}$ have more than one conjugacy class of subgroups isomorphic to $H_{[96,195]}$.

The shifts of

$$
\begin{gathered}
\Delta_{[96,195], 1}^{1}=1+a x a y w+a^{2} x a x a w+x w+x a^{2} y+a x a y+x a^{2} w+x a w+a^{2} x a x+ \\
a x a+a x w+a^{2} y+a x a^{2} x y w+a^{2} x a x y w+a x a x a x w+a x a^{2} x y+a x a x w+ \\
a^{2} x a+a^{2} x w+a x y w
\end{gathered}
$$

by $w$ and $a x a^{2} y w$ are equivalent regular PDS's. The corresponding SRG's with parameters $(96,20,4,4)$ are isomorphic to $\Gamma_{2}$.

The shift of

$$
\begin{gathered}
\Delta_{[96,195], 2}^{1}=1+x a x a w+a^{2} x a y w+a^{2} x a^{2} y+a x w+a^{2} x a x a x y w+a w+a x a^{2} y+ \\
a^{2} x a x y+x a^{2} x w+\text { axaxax }+a^{2} w+a^{2} x a x a+a^{2} x a x a y+a^{2} x a^{2}+ \\
w+a x a x w+a^{2} x a w+a^{2} x a x a y w+y w
\end{gathered}
$$

by $a^{2} w, e$ extracted, is a regular PDS. The corresponding SRG with parameters $(96,19,2,4)$ is isomorphic to $\Gamma_{1}$.

$\Delta_{[96,195], 2}^{1}$ multiplied by $x a^{2} y w$ is another regular PDS. The corresponding SRG with parameters $(96,20,4,4)$ we denote by $\Gamma_{3}$. Using GRAPE one finds $\left|A u t \Gamma_{3}\right|=1536$.

The shifts of

$$
\Delta_{[96,195], 3}^{1}=1+\operatorname{axax}+x a x y w+a^{2} x a x a+a^{2} y w+a x a y w+x a^{2} x w+y+x y+
$$




$$
\begin{aligned}
& x a x a x y+x+x a^{2}+a y+x a+a^{2} x a^{2} y+a^{2} x a^{2} x y w+a^{2} y+ \\
& \text { ayw }+x a x a x w+a^{2} x a x a y w
\end{aligned}
$$

by $a^{2} x a^{2} x y$ and $a x, e$ extracted, are equivalent regular PDS's. The corresponding SRG's with parameters $(96,19,2,4)$ are isomorphic to $\Gamma_{1}$.

Six regular PDS's are obtained starting from difference set

$\Delta_{[96,195], 4}^{1}=1+x a+$ axaxaw $+a^{2} x a x a w+x a^{2} x y+a x a+a^{2}+y+$

$x y+a^{2} x a x a+x+a^{2} x a^{2} y+x a x w+a w+a^{2} x a^{2} x w+$

$a^{2} x a y w+a y+x w+x a x a x y+a x a^{2} x y w$.

$\Delta_{[96,195], 4}^{1}$-shifts by $a^{2}, a x a^{2} x$, and $a^{2} x a x a$ are equivalent regular PDS's that correspond to SRG's with parameters $(96,20,4,4)$ isomorphic to $\Gamma_{3}$. On the other side, $\Delta_{[96,195], 4}^{1} x y \backslash\{e\}$, $\Delta_{[96,195], 4}^{1} x a^{2} y \backslash\{e\}$, and $\Delta_{[96,195], 4}^{1} x a y \backslash\{e\}$ are equivalent regular PDS's that correspond to SRG's with parameters $(96,19,2,4)$ isomorphic to $\Gamma_{1}$.

Six regular PDS's are obtained starting from difference set

$$
\begin{aligned}
& \Delta_{[96,195], 1}^{6}=1+a^{2} x a y+\text { axaxay }+a x a^{2}+a^{2}+a^{2} x y+a^{2} y+a^{2} x+a^{2} w+ \\
& x a x a w+\text { axaxw }+x a^{2} x w+a+x a+a^{2} x a^{2} x y+a^{2} x a x a x y+ \\
& \text { aw }+a^{2} x a^{2} y w+x a x y w+a^{2} x a x a x w .
\end{aligned}
$$

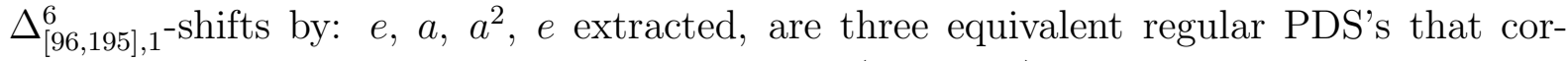
respond to isomorphic SRG's with parameters $(96,19,2,4)$. We denote them by $\Gamma_{4}$ and explore ([8]) Aut $\Gamma_{4}=[288,1026]$. Further three $\Delta_{[96,195], 1}^{6}$-shifts by $a^{2} x a^{2} y$, $a x a^{2} y$, and $x a^{2} y$ are equivalent regular PDS's corresponding to isomorphic SRG's with parameters $(96,20,4,4)$. We denote them by $\Gamma_{5}$ and using [8] find: $A u t \Gamma_{5}=[96,195]$.

Six regular PDS's are obtained starting from difference set

$$
\begin{gathered}
\Delta_{[96,195], 2}^{6}=1+a x a^{2}+a^{2} x a y+\text { axaxay }+a^{2} x a^{2} x+x a y+a y+a^{2} x a x a x+ \\
w+a x a^{2} y w+\text { axaxax } w+a^{2} x a x y w+x a x a+x a x a x y+ \\
\text { xaxax }+ \text { xaxay }+a^{2} x a^{2} x w+a^{2} x a x a w+x a x w+\text { aw } .
\end{gathered}
$$

$\Delta_{[96,195], 2}^{6}$-shifts by: $e, a^{2} x a^{2} x$, and $x a x a, e$ extracted, are equivalent regular PDS's that correspond to SRG's with parameters $(96,19,2,4)$ isomorphic to $\Gamma_{4}$. Further three $\Delta_{[96,195], 2^{-}}^{6}$ shifts by axay, $a x a^{2} y$, and $a x y$ are equivalent regular PDS's corresponding to three isomorphic SRG's with parameters $(96,20,4,4)$. We denote them by $\Gamma_{6} ; A u t \Gamma_{6}=[96,195]$. Nevertheless, $\Gamma_{5} \nsucceq \Gamma_{6}$.

Finally, two shifts of

$$
\begin{aligned}
\Delta_{[96,195]}^{8}= & 1+a x a^{2} x+\text { axaxa }+a^{2} x a x+a x a x+a^{2} x y+a^{2} x+\text { axaxy }+ \\
& a^{2} x a^{2} x w+a^{2} x a x a x w+a^{2} x x a x y w+a^{2} x a^{2} x y w+a^{2} x a x a+ \\
& a^{2} x a^{2} x y+a x+x a y+a^{2} w+x a x a x y w+a^{2} x w+x a x a y w
\end{aligned}
$$

are inequivalent regular PDS's. $\Delta_{[96,195]}^{8}$-shift by axaxaxyw corresponds to SRG with parameters $(96,20,4,4)$ isomorphic to $\Gamma_{7}$, while $\Delta_{[96,195]^{-}}^{8}$ shift by $a^{2} x a x w$ corresponds to SRG with parameters $(96,20,4,4)$ isomorphic to $\Gamma_{8}$.

Note that the group $H_{[96,195]}$ provides examples of mutually inequivalent regular PDS's that correspond to isomorphic strongly regular Cayley graphs. For instance, $\Delta_{[96,195], 2}^{1} a^{2} w$ $\backslash\{e\}, \Delta_{[96,195], 3}^{1} a x \backslash\{e\}$, and $\Delta_{[96,195], 4}^{1} x y \backslash\{e\}$ are isomorphic to $\Gamma_{1} ; \Delta_{[96,195], 1}^{6} \backslash\{e\}$ and $\Delta_{[96,195], 2}^{6} \backslash\{e\}$ are isomorphic to $\Gamma_{4}$. 
7. In the group:

$$
\begin{aligned}
H_{[96,197]}= & A_{4} \times D_{8}=\left\langle x, y, a \mid x^{2}=y^{2}=a^{3}=1, x^{a}=y, y^{a}=x y\right\rangle \times \\
& \times\left\langle z, t \mid z^{4}=t^{2}=(t z)^{2}=1\right\rangle
\end{aligned}
$$

one difference set enables construction of, up to equivalency, one regular PDS.

The shifts of

$$
\begin{aligned}
\Delta_{[96,197]}^{1} & =1+a^{2}+a^{2} x a z^{3}+a^{2} x z^{3}+a^{2} x a^{2} z^{3}+a^{2} x a^{2} z+a z+x z^{2}+a^{2} x a z t+ \\
& a^{2} x z^{3} t+a x a^{2} z^{3} t+x a^{2} z t+x a^{2} z^{3}+a^{2} x z^{2} t+x a^{2} z^{2} t+a x a^{2} t+a x a z^{2}+ \\
& a x a^{2} z^{3}+a z^{3}+a^{2} x a t
\end{aligned}
$$

by $a^{2} z^{3} t$ and $a^{2} z t$ are equivalent regular PDS's. Two corresponding SRG's with parameters $(96,20,4,4)$ are isomorphic to $\Gamma_{2}$.

8. In the group:

$$
\begin{aligned}
H_{[96,226]}= & S_{4} \times Z_{2}^{2}=\langle x, y, a| x^{2}=y^{2}=[x, y]=a^{3}=(x y)^{3}=(y a)^{2}=1, \\
& \left.(x y a)^{2}=1\right\rangle \times\left\langle z, t \mid z^{2}=t^{2}=[z, t]=1\right\rangle
\end{aligned}
$$

the difference set

$$
\begin{aligned}
\Delta_{[96,226]}^{1} & =1+x t+x a^{2}+x a x z t+x a z+x a^{2} x a x z+x a+a x a x z t+a^{2} x a^{2} x z+ \\
& a x a t+a x a^{2} x a t+a x a+a^{2} z+x a^{2} x a+a x a^{2} x t+a^{2} x a+x+x a^{2} z t+ \\
& x a^{2} x a x+a^{2} x a z t
\end{aligned}
$$

enables construction of, up to equivalency, one regular PDS. The $\Delta_{[96,226]}^{1}$-shifts by $x a^{2} x a$, $x a^{2} x a z, x a^{2} x a t$, and $x a^{2} x a z t$ are mutually equivalent regular PDS's. The corresponding SRG's with parameters $(96,20,4,4)$ are isomorphic to $\Gamma_{2}$.

9. In the group:

$$
\begin{gathered}
H_{[96,227]}=\langle x, y, z, t, w, a| x^{2}=y^{2}=z^{2}=t^{2}=[x, y]=[x, z]=[x, t]=[y, z]=1, \\
{[y, t]=[z, t]=1, w^{2}=a^{3}=(w a)^{2}=1, x^{w}=y, y^{w}=x, z^{w}=t,} \\
\left.t^{w}=z, x^{a}=x y, y^{a}=x, z^{a}=t, t^{a}=z t\right\rangle
\end{gathered}
$$

four inequivalent difference sets originating from three nonisomorphic symmetric designs enable construction of 6 inequivalent regular PDS's. Aut $D_{1}$ has more than one conjugacy class of subgroups isomorphic to $H_{[96,227]}$.

The shift of

$$
\begin{gathered}
\Delta_{[96,227], 1}^{1}=1+z a^{2} w+x a z a^{2} w+x a+x a^{2} z a^{2} w+a x w+a x a^{2} z a w+a x a^{2}+ \\
a^{2} x a+x a^{2} z w+x+a x z a w+a^{2} x a^{2} z+x a^{2} z a^{2}+a^{2} x a z a+ \\
a^{2} z a w+a^{2} x w+z w+x a z w+x w
\end{gathered}
$$

by $a x a^{2} z a^{2} w$ is a regular PDS. It corresponds to SRG with parameters $(96,20,4,4)$ isomorphic to $\Gamma_{3}$. Another regular PDS can be obtained as $\Delta_{[96,227], 1}^{1}$-shift by $a^{2} x w$ and $e$ extracted. The corresponding SRG with parameters $(96,19,2,4)$ is isomorphic to $\Gamma_{1}$.

The shifts of

$$
\begin{gathered}
\Delta_{[96,227,2}^{1}=1+x a z a+a x a^{2} z a w+x w+a x a z a^{2}+a^{2} z a^{2}+a^{2} x a^{2}+x z+ \\
a x a^{2} z+a x a+a^{2} x a+a z a+a z a^{2} w+a^{2} x z a w+a x a^{2} z w+ \\
a x a z a^{2} w+a^{2} x+a x w+x a+a x z w
\end{gathered}
$$

by $a^{2} x a, a^{2} x a^{2}, a^{2} x, a^{2} z a^{2}, a x a^{2} z$, and $x a z a, e$ extracted, are equivalent regular PDS's. The corresponding six SRG's with parameters $(96,19,2,4)$ are isomorphic to $\Gamma_{1}$. 
The shifts of

$$
\begin{aligned}
\Delta_{[96,227]}^{6} & =1+a x a^{2} z+x z+a^{2} x a+a x a+a^{2} x+x a z a+a z a+a x a^{2} w+ \\
& a x a z a w+a x z a^{2} w+a x a^{2} z w+a^{2} x a^{2}+a x a z a^{2}+x a+ \\
& a^{2} z a^{2}+a^{2} x w+a x z a w+z a^{2} w+x a^{2} z w
\end{aligned}
$$

by $e, a^{2} x a^{2}$, and $a x a$, then $e$ extracted, are three equivalent regular PDS's that correspond to $\mathrm{SRG}$ 's with parameters $(96,19,2,4)$ isomorphic to $\Gamma_{4}$.

The shift of

$$
\begin{gathered}
\Delta_{[96,227]}^{8}=1+z+a z a^{2}+a^{2} z a+a^{2} x a^{2} z+x z a+a^{2} z a^{2}+a x+a z w+ \\
a x z w+x z a w+a^{2} x a z a w+a x a^{2} w+a^{2} x a z w+z w+x w+ \\
a x a^{2} z a^{2}+x a^{2} z+a^{2} z+a^{2} x a z a^{2}
\end{gathered}
$$

by $a^{2} x a z a^{2} w$ is a regular PDS corresponding to SRG with parameters $(96,20,4,4)$ isomorphic to $\Gamma_{8} . \Delta_{[96,227]^{-}}^{8}$-shift by azaw is a regular PDS corresponding to SRG with parameters $(96,20,4,4)$ isomorphic to $\Gamma_{7}$.

In $H_{[96,227]}$ we have again the case of two mutually inequivalent regular partial difference sets that correspond to isomorphic strongly regular Cayley graphs.

Regarding the obtained SRG's with 96 vertices, our results can be summarized in (2.1).

\begin{tabular}{c|c|c|c|c} 
Graph & Valency & Corresp. design & $\left|A u t \Gamma_{i}\right|$ & Vertex group id. number \\
\hline \hline$\Gamma_{1}$ & 19 & $D_{1}$ & 9216 & {$[64 ; 70 ; 71 ; 195 ; 227]$} \\
$\Gamma_{2}$ & 20 & $D_{1}$ & 11520 & {$[186 ; 190 ; 195 ; 197 ; 226]$} \\
$\Gamma_{3}$ & 20 & $D_{1}$ & 1536 & {$[195 ; 227]$} \\
\hline$\Gamma_{4}$ & 19 & $D_{6}$ & 288 & {$[195 ; 227]$} \\
$\Gamma_{5}$ & 20 & $D_{6}$ & 96 & {$[195]$} \\
$\Gamma_{6}$ & 20 & $D_{6}$ & 96 & {$[195]$} \\
\hline$\Gamma_{7}$ & 20 & $D_{8}$ & 3072 & {$[70 ; 190 ; 195 ; 227]$} \\
$\Gamma_{8}$ & 20 & $D_{8}$ & 138240 & {$[64 ; 70 ; 71 ; 190 ; 195 ; 227]$}
\end{tabular}

Six graphs are with parameters $(96,20,4,4)$ and two with parameters $(96,19,2,4)$. Two of the obtained graphs proved to be isomorphic to the already known graphs. Up to isomorphism (GRAPE-tested), $\Gamma_{8}$ is the collinearity graph of $G Q(5,3)$ and $\Gamma_{2}$ is the graph denoted by $K^{\prime}$ in [2]. Note that each $\Gamma_{j}, j=1,2, \ldots, 8$ can be represented as a PDS in the group $H_{[96,195]}$. By single horizontal lines in (2.1) a kind of "design-equivalence" is framed, but this, of course, regards only parameters $(96,20,4,4)$. The GRAPE-files determining $\Gamma_{i}, i=1,2, \ldots, 8$ are available at http://www.pmfst.hr/ ${ }^{\sim}$ vucicic/8srg96v.txt.

\section{References}

[1] T. Beth, D. Jungnickel, and H. Lenz, Design theory, Cambridge University Press (1999). 
[2] A.E. Brouwer, J.H. Koolen and M.H. Klin, A Root Graph That is Locally the Line Graph of the Petersen Graph, Discrete Mathematics 264 (2003), 13-24.

[3] A. Golemac, J. Mandić and T. Vučičić, On the Existence of Difference Sets in Groups of Order 96, to appear in Discrete Mathematics.

[4] A. Golemac, J. Mandić and T. Vučičić, One $(96,20,4)$ Symmetric Design and Related Nonabelian Difference Sets, Designs, Codes and Cryptography, 37 (2005), 5-13.

[5] L.K. Jorgensen and M.H. Klin, Switching of edges in strongly regular graphs. I.: A family of partial difference sets on 100 vertices, The Electronic Journal of Combinatorics, 1077-8926, vol. 101 ed. (2003), 17-31.

[6] S.L. Ma, Partial Difference Sets, Discrete Mathematics, 52 (1984), 75-89.

[7] S.L. Ma, A Survey of Partial Difference Sets, Designs, Codes and Cryptography, 4 (1994), 221-261.

[8] L.H. Soicher, GRAPE: a system for computing with graphs and groups. In: Groups and computations (eds. Finkelstein and Kantor), volume 11 of DIMACS Series in Discrete Mathematics and Theoretical Computer Science, AMS, 1993, 287-291.

[9] [GAP 99] The GAP Group, GAP - - - Groups, Algorithms, and Programming, Version 4.3; Aachen, St. Andrews (1999). 